\title{
Strain sensor based on hollow microsphere Fabry-Perot cavity
}

\author{
Catarina S. Monteiro ${ }^{a}$, Susana O. Silva ${ }^{\mathrm{a}}$, Orlando Frazão ${ }^{\mathrm{a}^{*}}$ \\ ${ }^{a}$ INESC TEC and Department of Physics and Astronomy, Faculty of Sciences of University of Porto, \\ Rua do Campo Alegre 687, 4169-007 Porto, Portugal
}

\begin{abstract}
Fusion splicing technique was explored for the fabrication of two sensing structures based on hollow microsphere FabryPerot cavity. The first sensor proposed was fabricated with a hollow microsphere tip, working as a probe sensor. This structure was studied for lateral load pressure, yielding a $1.56 \pm 0.01 \mathrm{~nm} / \mathrm{N}$ sensitivity. The second sensing structure relied on an in-line hollow microsphere, which allowed the detection of lateral load, with a sensitivity of $2.62 \pm 0.02 \mathrm{~nm} / \mathrm{N}$. Furthermore, the proposed structure enabled strain sensing, with a sensitivity of $4.66 \pm 0.03 \mathrm{pm} / \mu \varepsilon$. The two sensing structures were subjected to temperature, presenting low thermal cross-sensitivity.
\end{abstract}

Keywords: Fabry-Perot interferometry, Fiber optics, Lateral loading, Optical sensors, Strain, Temperature

\section{INTRODUCTION}

Interferometric configurations applied to optical fiber as sensors have been widely researched and developed in the last years. In particular, the low finesse Fabry-Perot presents itself as a great solution for sensing several physical and chemical parameters. The interferometric pattern that this configuration presents results from the interference between two or more reflected waves with different optical paths. The fabrication of optical fiber sensors based on the low finesse Fabry-Perot interferometer have been achieved by metal deposition ${ }^{1}$, chemical etching ${ }^{2}$, by micromachining ${ }^{3}$, and simply by fusion splicing ${ }^{4}$. Although all fabrication techniques present advantages for different applications, fusion splicing is a simple, fast, and highly reproducible technique that does not present chemical hazards which makes it ideal for the fabrication of microsphere based sensors. Microsphere cavities have been proposed by Villatoro et al., by using an arc discharge to form an air bubble between a standard single mode fiber (SMF) and a photonic crystal fiber ${ }^{5}$. The use of capillary fiber of silica ${ }^{6}$ and standard $\mathrm{SMF}^{7,8}$ for the fabrication of a hollow microsphere cavity were also demonstrated.

In this work, two different sensing structures based on a hollow microsphere Fabry-Perot cavity are demonstrated. The two sensing configurations are fabricated relying only on the fusion splice technique. The first proposed sensor has a probe configuration, with one hollow microsphere at the tip of the fiber. The second sensor demonstrated has an in-line configuration with one microsphere cavity. The two structures are subjected to lateral loading studies, demonstrating similar responses. The in-line configuration allows strain sensing, achieving high sensitivity. Both sensor structures were tested for temperature variations, showing low thermal cross-sensitivity to the further measurands.

\section{SENSOR FABRICATION AND OPERATION PRINCIPLE}

\subsection{Sensor fabrication}

The fabrication process of the two sensing structures is schematized in fig. 1, and the fusion splice programs parameters are presented in Table 1. The splicing machine used in the fabrication process was a Sumitomo 71C. The process starts with fusion splicing a cleaved SMF to a hollow core fiber, as presented in fig. 1(a), by using the manual Program 1 in Table 1. The center of the electric arc is set on the SMF to prevent the collapse of the hollow core fiber, while being able to join the two fibers. After cleaving the hollow fiber, two consecutive electric arcs are applied at the tip of the fiber, by using the manual Program 2 (Table 1), to form a thinner microsphere cavity, as shown in fig. 1(c). The resultant structure

* orlando.frazao@inesctec.pt, Telephone: +351220402301 
is a Fabry-Perot interferometer with a microsphere cavity, as presented in fig. 1(e). The fabricated sensor showed an air cavity of $\sim 175 \mu \mathrm{m}$ and a silica wall with a $\sim 30 \mu \mathrm{m}$ width. For the in-line configuration the manual Program 3 of Table 1 was used to join one tip structure previously fabricated to a cleaved SMF, resulting in an $\sim 160 \mu \mathrm{m}$ optical cavity as presented in fig. $1(\mathrm{f})$.

Table 1. Manual splicing parameters.

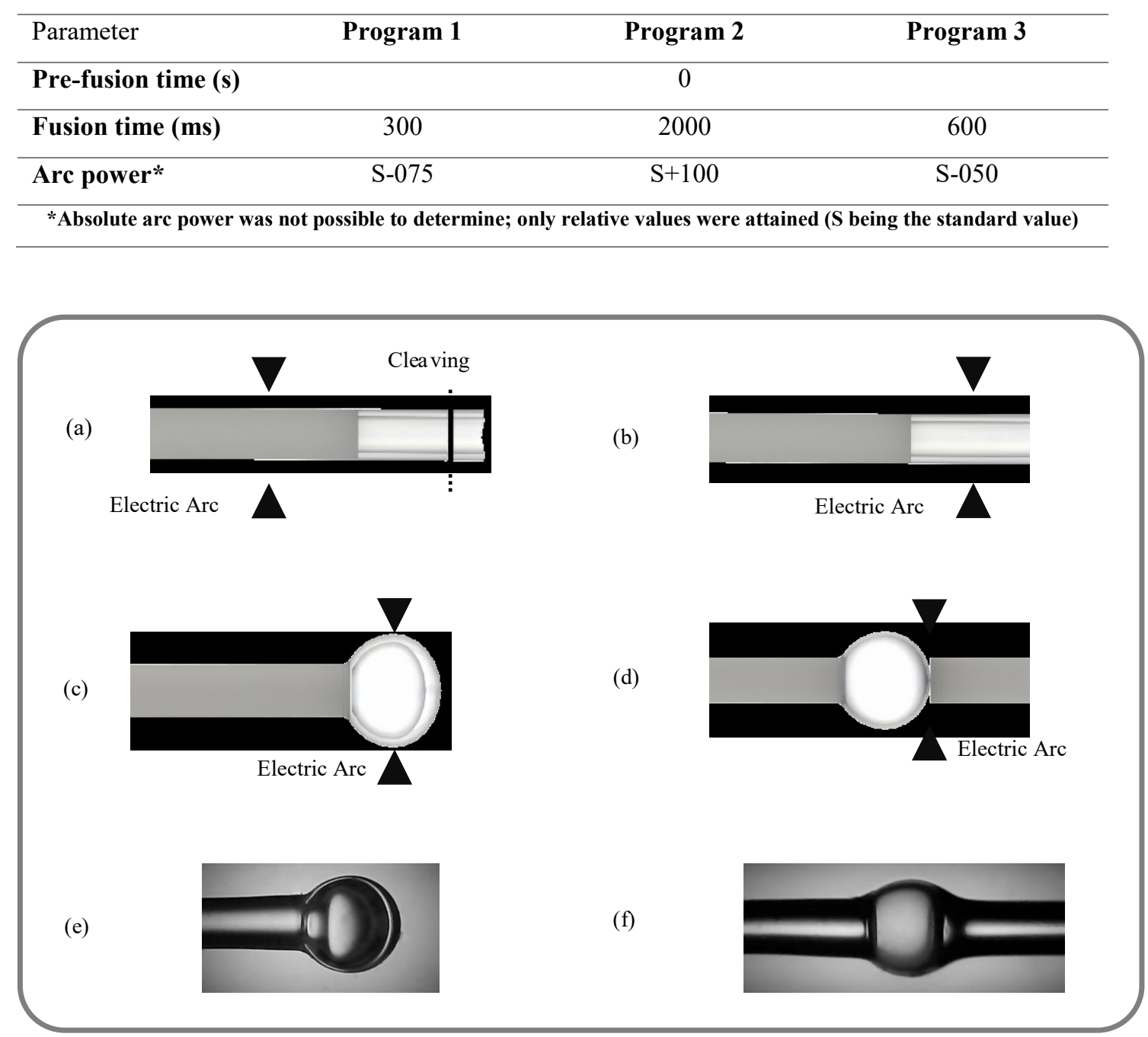

Figure 1. Fabrication process of in tip sensor (a-c) and the in-line sensor (a-d). Microscope photograph of (e) the tip sensor and (f) the in-line sensor.

\subsection{Operation principle}

The spectrum of the tip sensor is the result from the interference of three waves with different optical paths. The three waves result from the reflection at the interfaces with different refractive indexes: the silica/air, air/silica and the silica/surrounding media interfaces. The dominant interferences are between wave 1 and 2 and between wave 1 and 3 , as the interference between the second and third reflected waves can be neglected due to the small width of the microsphere. These interferences result in a beat as presented in fig. 2(a). The in-line structure presents a much simpler spectrum 
considering that it results from the interference of two waves, making this configuration a two-wave interferometer as shown in fig. 2(b).

(a)

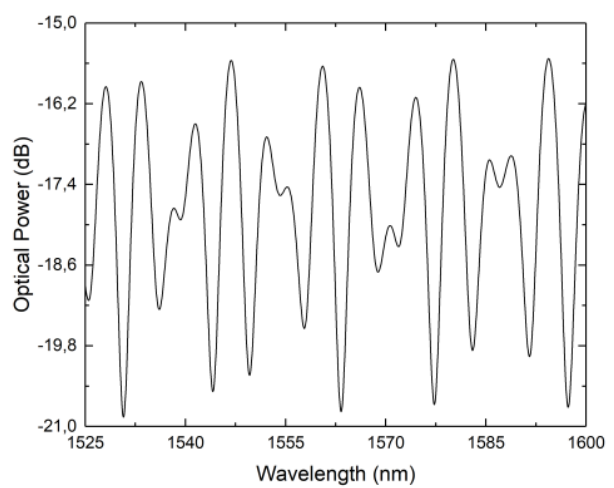

(b)

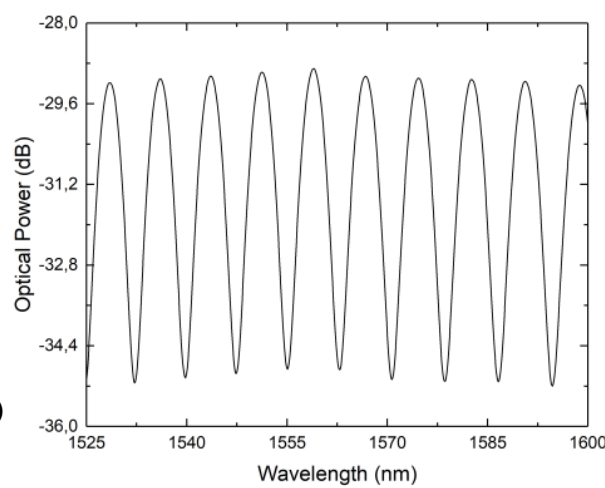

Figure 2. Reflection spectrum of (a) tip sensor and (b) in-line sensor.

\section{EXPERIMENTAL RESULTS}

The interrogation system was composed by a broadband optical source, centered at $1550 \mathrm{~nm}$ with a bandwidth of $100 \mathrm{~nm}$, and optical circulator and an optical spectrum analyzer (OSA). The spectra of the sensors were attained in reflection. The two sensors were tested for lateral load and temperature. The in-line structure enables the detection of strain and was also tested for this measurand.

Lateral load tests were carried out for the two sensors for loads that ranged from 0 to $\sim 1.4 \mathrm{~N}$. The variation of load resulted in a wavelength shift in the spectra of the sensors, as presented in fig. 3(a). A sensitivity of $1.56 \pm 0.01 \mathrm{~nm} / \mathrm{N}$ was achieved for the tip sensor, presenting a result consistent to previous works in the literature ${ }^{6}$. Regarding the in-line structure, a sensitivity of $2.62 \pm 0.02 \mathrm{~nm} / \mathrm{N}$ was achieved. The higher sensitivity may be due to the thinner walls achieved in the fabricated sensor. The temperature response for the two sensors has linear behavior in the tested range. The tip sensor was tested for temperatures between 300 and $500^{\circ} \mathrm{C}$, resulting in a $1.90 \pm 0.06 \mathrm{pm} /{ }^{\circ} \mathrm{C}$ sensitivity with a cross-sensitivity of $1 \times 10^{-3} \mathrm{~N} /{ }^{\circ} \mathrm{C}$. The in-line configuration attained a $1.17 \pm 0.03 \mathrm{pm} /{ }^{\circ} \mathrm{C}$ sensitivity for temperatures ranging from 250 to $600{ }^{\circ} \mathrm{C}$, with a cross-sensitivity of $0.75 \times 10^{-3} \mathrm{~N} /{ }^{\circ} \mathrm{C}$.

The in-line configuration was tested for strain up to $1000 \mu \varepsilon$, as shown in fig. 3(b). The sensor presented a sensitivity of $4.66 \pm 0.03 \mathrm{pm} / \mu \varepsilon$, with a cross-sensitivity of $0.25 \mu \varepsilon /{ }^{\circ} \mathrm{C}$ to temperature. The achieved sensitivity is almost three times higher than that proposed in the literature ${ }^{\text {? }}$.

(a)

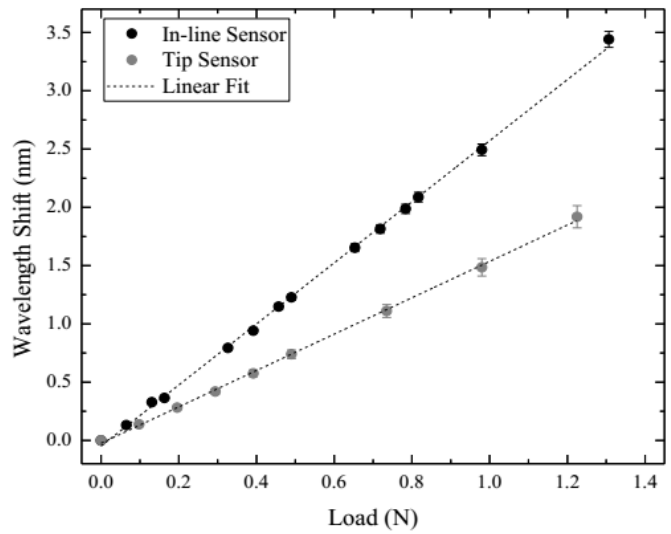

(b)

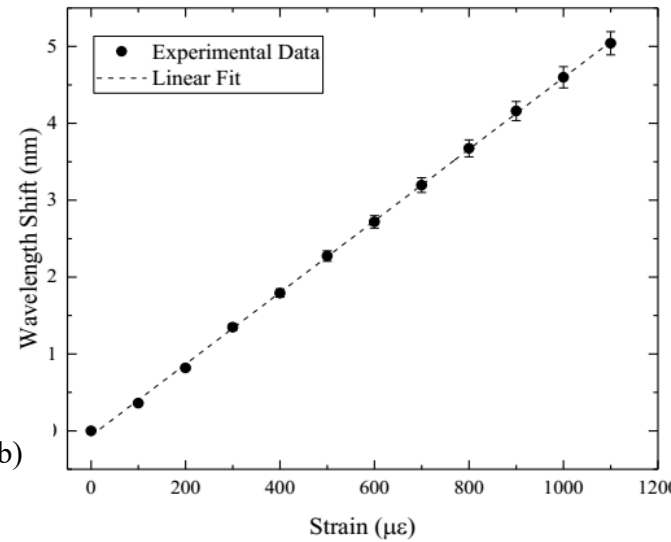

Figure 3. (a) Lateral loading response for in-line sensor and tip sensor. (b) Strain response for in-line sensor. 


\section{CONCLUSIONS}

Two sensing structures with a hollow microsphere based on Fabry-Perot interferometry were proposed. The two configurations were tested for lateral loading and temperature attaining comparable results in both structures. The first presented sensor, with a tip configuration, showed a $1.56 \pm 0.01 \mathrm{~nm} / \mathrm{N}$ sensitivity for lateral load tests with a crosssensitivity of $1 \times 10^{-3} \mathrm{~N} /{ }^{\circ} \mathrm{C}$ to temperature. The second proposed structure, an in-line sensor, yielded a $2.62 \pm 0.02 \mathrm{~nm} / \mathrm{N}$ sensitivity with a cross-sensitivity of $0.75 \times 10^{-3} \mathrm{~N} /{ }^{\circ} \mathrm{C}$ to temperature. The higher sensitivity for this measurand may be due to the thinner walls presented in this structure when compared to the microsphere of the tip sensor. The in-line configuration allowed strain sensing, for a maximum of $1000 \mu \varepsilon$, attaining a sensitivity of $4.66 \pm 0.03 \mathrm{pm} / \mu \varepsilon$, a sensitivity around 3 times higher than that proposed in the literature. The two proposed structures may be suitable for lateral loading sensing in harsh environments. The in-line configuration may be also suited for strain sensing under harsh conditions.

\section{ACKNOWLEDGMENTS}

CORAL - Sustainable Ocean Exploitation: Tools and Sensors, NORTE-01-0145-FEDER-000036, financed by the North Portugal Regional Operational Programme (NORTE 2020), under the PORTUGAL 2020 Partnership Agreement, and through the European Regional Development Fund (ERDF).

\section{REFERENCES}

1. Xu F, Ren D, Shi X, et al. High-sensitivity Fabry-Perot interferometric pressure sensor based on a nanothick silver diaphragm. Opt Lett. 2012;37(2):133. doi:10.1364/OL.37.000133.

2. Machavaram VR, Badcock RA, Fernando GF. Fabrication of intrinsic fibre Fabry-Perot sensors in silica fibres using hydrofluoric acid etching. Sensors Actuators, A Phys. 2007;138(1):248-260. doi:10.1016/j.sna.2007.04.007.

3. André RM, Warren-Smith SC, Becker M, et al. Simultaneous measurement of temperature and refractive index using focused ion beam milled Fabry-Perot cavities in optical fiber micro-tips. Opt Express. 2016;24(13):14053. doi:10.1364/OE.24.014053.

4. Sirkis J, Jones RT, Singh H, et al. In-line fiber etalon (ILFE) fiber-optic strain sensors. J Light Technol. 1995;13(7):1256-1263. doi:10.1109/50.400690.

5. Villatoro J, Finazzi V, Coviello G, Pruneri V. Photonic-crystal-fiber-enabled micro-Fabry-Perot interferometer Opt Lett. 2009;34(16):2441-2443. doi:10.1364/OL.34.002441.

6. Ma J, Ju J, Jin L, Jin W, Wang D. Fiber-tip micro-cavity for temperature and transverse load sensing. Opt Express. 2011;19(13):12418-12426. doi:10.1364/OE.19.012418.

7. Liu S, Wang YP, Liao CR, et al. High-sensitivity strain sensor based on in-fiber improved Fabry-Perot interferometer. Opt Lett. 2014;39(7):2121-2124. doi:Doi 10.1364/O1.39.002121.

8. Liao C, Liu S, Xu L, et al. Sub-micron silica diaphragm-based fiber-tip Fabry-Perot interferometer for pressure measurement. Opt Lett. 2014;39(10):2827. doi:10.1364/OL.39.002827.

9. $\quad$ Ferreira MS, Santos JL, Frazão O. Silica microspheres array strain sensor. Opt Lett. 2014;39(20):5937-5940. doi:10.1364/OL.39.005937. 